\title{
Weak Invertibility of Finite Automata and Cryptanalysis on FAPKC*
}

\author{
ZongDuo Dai ${ }^{1}$, Ding Feng $\mathrm{Ye}^{2}$ and Kwok Yan Lam $^{2}$ \\ ${ }^{1}$ Dept. of Math., State Key Lab. of Information Security \\ Graduate School, Academia Sinica, 100039-08, Beijing, China, \\ yangdai@mimi.cnc.ac.cn \\ ${ }^{2}$ Dept. of ISCS, National University of Singapore, \\ Lower Kent Ridge Road, Singapore 119260 \\ yedf@iscs.nus.sg lamky@iscs.nus.sg
}

\begin{abstract}
FAPKC $[17,18,19,20,22]$ is a public key cryptosystem based weakly invertible finite automata. Weak invertibility of FAs is the key to understand and analyze this scheme. In this paper a set of algebraic terminologies describing FAs is developed, and the theory of weak invertibility of FAs is studied. Based on this, a cryptanalysis on FAPKC is made. It is shown that the keys proposed in $[17,18,19,20,21]$ for FAPKCs are insecure both in encrypting and in signing.
\end{abstract}

Keywords: finite automaton, public key cryptosystem, cryptanalysis

\section{Introduction}

Finite automaton (FA) is a widely used concept in computer science and has several definitions slightly different to each other according to applications. In this context, it refers to a finite sequential state machine, which was studied widely, say for example in [1-16]. The action of such a machine is controlled by a clock which ticks with inputs, i.e., on receiving an input symbol, it produces an output symbol and its state transfers to a new one according to certain rules, and thus with an initial state and an input sequence of finite length it produces an output sequence of the same length. Hence a finite automaton is an analogue to a usual function when viewed as a transformation from input sequences to output sequences. A weakly invertible finite automaton (WIFA) with delay $\tau$, or simply $\tau$-weakly invertible finite automaton, is such a FA that any input is uniquely determined by the corresponding state and output together with the subsequent $\tau$ outputs. That is, the input information can be recovered from the outputs after waiting $\tau$ steps, or in other words, with $\tau$ delays. WIFAs are similar to the usual injective functions in the respect that one can retrieve the input

*This work was supported by Chinese Natural Science Foundation. 
information from the outputs. However the delay $\tau$ and the state dependence make it much more complicated for one to recover the input information than the usual injective functions. The first objective of this paper is to set up a systematic theory in dealing with the the problems such as how to construct weak invertible FAs and their weak inverses, and how to routinely retrieve input information from outputs and the initial state.

FAPKC, which is a public key cryptosystem and can do both encrypting and signing, is based on weakly invertible finite automata (WIFAs). FAPKC was first introduced in 1985 [17], named as FAPKC0. Some versions were published in 1986 [18], named as FAPKC1 and FAPKC2. Then a new version was introduced in 1995 [20], named as FAPKC3. Roughly speaking, in all these systems, the private key consists of two FAs whose weak inverses can be easy constructed, and the public key is the composition of them. It is believed in [18-21] that it is hard to decompose the public key to get the private two FAs and that it is hard to get a weak inverse of the composed FA without knowing this decomposition, hence any user can encrypt messages or verify signatures using the public key, but can neither decrypt the cipher-texts nor forge the signatures without knowing its two private components. To hide the components from the composed FA, it is proposed to use boolean functions to express the composition. Then how to maintain a moderate public key size becomes a big problem, as composition would generally yield boolean expression exploding when the outer component is nonlinear. The proposed method is to restrict the input set $X$ equal or smaller than $F^{8}$, where $F$ is the binary field $G F(2)$, and to restrict the nonlinear degree of the components to be small. The early versions were analysed in some papers, say in $[23,24,25,26]$.

The main contribution of this paper consists of two parts. In the first part (Section 3-5), we develop a set of algebraic terminologies to describe FAs and give a systematic treatment to the weak invertibility theory on the seperable memory FAs. In the second part (Section 6-7), based on the developed theory, we make a simple introduction to FAPKC and then a cryptanalysis on it. Our results show that all the keys proposed for FAPKC in $[17,18,19,20,21]$ are insecure both in encrypting and in signing. Before coming to the main topic, we recall some basic definitions in the next section.

Due to lack of space, the proofs of all the lemmas and theorems in this paper are ommited.

\section{Basic Definitions}

For convenience, in this section we restate some basic concepts, which can be found in $[8]$ except some concepts like the natural pairs and the right $\tau$-weak inverses.

A finite automaton (FA) is a pentad $M=(X, Y, S, \delta, \lambda)$ where $X, Y$ are input and output symbol sets respectively, $S$ is the state set, $X, Y$ and $S$ are all finite, $\delta: S \times X \rightarrow S$ is the next state function, and $\lambda: S \times X \rightarrow Y$ is the output function. In the sequel, let $X^{i}=\left\{x_{0} x_{1} \cdots x_{i-1} \mid x_{j} \in X, 0 \leq j<i\right\}$ be the set 
of all input sequence of length $i$, similarly for $Y^{i}$. For any $s \in S$, we use $M(s)$ and $\delta(s)$ denote the function from $\bigcup_{i \geq 1} X^{i}$ to $\bigcup_{i \geq 1} Y^{i}$ and the function from $\bigcup_{i \geq 1} X^{i}$ to $S$ defined as

$$
\begin{aligned}
M(s) x_{0} x_{1} \cdots x_{i-1} & =y_{0} y_{1} \cdots y_{i-1} \\
\delta(s) x_{0} x_{1} \cdots x_{i-1} & =s_{i}
\end{aligned}
$$

, where $s_{0}=s, s_{j+1}=\delta\left(s_{j}, x_{j}\right), y_{j}=\lambda\left(s_{j}, x_{j}\right), x_{j} \in X, 0 \leq j<i$. For any two FAs $M, M^{\prime}$ which have the same input space $X$ and the same output space $Y$, we say a state $s$ in $M$ is equivalent to a state $s^{\prime}$ in $M^{\prime}$ if $M(s)=M^{\prime}\left(s^{\prime}\right)$, denoted by $s \sim s^{\prime}$; we say $M$ is a $s u b$-automaton of $M^{\prime}$, denoted by $M \leq M^{\prime}$, if for any state $s$ in $M$ there exists a state $s^{\prime}$ such that $s \sim s^{\prime}$; we say $M$ and $M^{\prime}$ are equivalent if $M \leq M^{\prime} \leq M$. We do not distinguish equivalent FAs in the rest of this paper.

A FA $M$ is called $\tau$-weakly invertible, if for any $s \in S, x_{i}, x_{i}^{\prime} \in X$, the following condition

$$
M(s) x_{0}^{\prime} x_{1}^{\prime} \cdots x_{\tau}^{\prime}=M(s) x_{0} x_{1} \cdots x_{\tau}
$$

implies $x_{0}^{\prime}=x_{0}$. The least such $\tau$, denoted by $\tau(M)$, is called information delay of $M$.

Let $M_{1}=\left(X, Y, S_{1}, \delta_{1}, \lambda_{1}\right)$ and $M_{2}=\left(Y, Z, S_{2}, \delta_{2}, \lambda_{2}\right)$ be two FAs, define the composition of $M_{2}$ and $M_{1}$ to be the FA $M_{2} \times M_{1}=\left(X, X, S_{2} \times S_{1}, \delta_{2} \times \delta_{1}\right.$, $\lambda_{2} \times \lambda_{1}$ ) where

$$
\begin{aligned}
\left(\lambda_{2} \times \lambda_{1}\right)\left(\left(s_{2}, s_{1}\right), x\right) & =\lambda_{2}\left(s_{2}, \lambda_{1}\left(s_{1}, x\right)\right) \\
\left(\delta_{2} \times \delta_{1}\right)\left(\left(s_{2}, s_{1}\right), x\right) & =\left(\delta_{2}\left(s_{2}, \lambda_{1}\left(s_{1}, x\right)\right), \delta_{1}\left(s_{1}, x\right)\right)
\end{aligned}
$$

$\forall\left(s_{2}, s_{1}\right) \in S_{2} \times S_{1}, x \in X$; we usually call $M_{1}$ the inner component, $M_{2}$ the outer component. It is true that $\left(M_{2} \times M_{1}\right)\left(s_{2}, s_{1}\right)=M_{2}\left(s_{2}\right) M_{1}\left(s_{1}\right)$.

Let $M=(X, Y, S, \delta, \lambda)$ and $M^{*}=\left(Y, X, S^{*}, \delta^{*}, \lambda^{*}\right)$ be two FAs. For $s \in$ $S, s^{*} \in S^{*}$, we say $\left(s^{*}, s\right)$ is a $\tau$-pair in $M^{*} \times M$, or $s^{*}$ is a left $\tau$-match of $s$, or $s$ is a right $\tau$-match of $s^{*}$, if

$$
\left(M^{*} \times M\right)\left(s^{*}, s\right) x_{0} x_{1} \cdots x_{n+\tau-1}=w_{0} w_{1} \cdots w_{\tau-1} x_{0} x_{1} \cdots x_{n-1}
$$

for all $x_{0} x_{1} \cdots x_{n+\tau-1} \in X^{n+\tau}$, where $w_{0} w_{1} \cdots w_{\tau-1} \in X^{\tau}$ may dependent on $x_{0} x_{1} \cdots x_{\tau-1}$. If further $w_{0} w_{1} \cdots w_{\tau-1}$ is independent on $x_{0} x_{1} \cdots x_{\tau-1}$, we say that $\left(s^{*}, s\right)$ is a natural $\tau$-pair.

Let $M$ and $M^{*}$ be as above, $M^{*}$ is called a $\tau$-weak inverse of $M$ and $\tau$ is called the recovery delay of $M^{*}$ (with respect to $M$ ), if for any $s \in S$, there exists a $s^{*} \in S^{*}$, such that $\left(s^{*}, s\right)$ is a $\tau$-pair in $M^{*} \times M$. It is clear that a $\tau$-weak inverse of $M$ can recover the input sequence except the last $\tau$ inputs.

In studying the commutability of a FA $M$ and its a weak inverse, we introduce the so-called right weak inverse of $M$. A FA $M^{*}$ is called a right $\tau$-weak inverse of $M$, if for any state $s$ in $M$, there exists a state $s^{*}$ in $M^{*}$, such that $\left(s, s^{*}\right)$ is a $\tau$-pair in $M \times M^{*}$. 


\section{$3 \quad$ Input Memory FAs and Quasi-Ring $\mathcal{F}$}

From now on, we assume $X=Y=F^{l}$ (elements being written as column vectors), where $F=G F(2)$ is the binary field, though all the results in this paper hold true when $F$ is any finite field. We will concentrate on the so called input memory FAs whose states are determined by some number of the past inputs (see below for the exact definition). Instead of investigating these FAs individually, we study them as a whole set (the quasi-ring $\mathcal{F}$ ) endowed with some algebraic structure. That is essential to our understanding of FAs. We begin with some definitions.

Let $\beta=\beta\left(t_{-h}, \cdots, t_{0}, u_{-k}, \cdots, u_{-1}\right)$ be a function: $X^{1+h} \times Y^{k} \rightarrow Y$. Define the memory order of $\beta$ to be the minimal integer pair $\left(h^{\prime}, k^{\prime}\right)$ such that $\beta$ is irrelevant to all the variables $\left\{t_{-i}, u_{-j} \mid i>h^{\prime} \geq-1, j>k^{\prime} \geq-1\right\}$, and denote it by $m(\beta)=\left(h^{\prime}, k^{\prime}\right)$.

This function $\beta$ together with any integer pair $(h, k), h \geq h^{\prime}, k \geq k^{\prime}$, determines a memory FA $M(\beta)^{(h, k)}=\left(X, X, S_{\beta}=X^{h} \times Y^{k}, \delta_{\beta}, \lambda_{\beta}\right)$ of type $(h, k)$, where for any state $s_{0}=\left(x_{-h} \cdots x_{-1}, y_{-k} \cdots y_{-1}\right) \in X^{h} \times X^{k}$, which is made of the past $h$ inputs and the past $k$ outputs, and any input $x_{0} \in X$,

$$
\begin{aligned}
\lambda_{\beta}\left(s_{0}, x_{0}\right) & =\beta\left(x_{-h}, \cdots, x_{-1}, x_{0}, y_{-k}, \cdots, y_{-1}\right) \\
\delta_{\beta}\left(s_{0}, x_{0}\right) & =\left(x_{-h+1} \cdots x_{0}, y_{-k+1} \cdots y_{-1} \lambda_{\beta}\left(s_{0}, x_{0}\right)\right)
\end{aligned}
$$

Notice that all the FAs $M(\beta)^{(h, k)}, h \geq h^{\prime}, k \geq k^{\prime}$, are equivalent to each other, so we do not care the type $(h, k)$, and write them by the same notation $M(\beta)$, or simply by $\beta$ when there is no ambiguity.

If the function $\beta$ is of the form

$$
\beta=f\left(t_{-h}, \cdots, t_{0}\right)+g\left(u_{-k}, \cdots, u_{-1}\right)
$$

we say $M(\beta)$ is a separable memory $F A$, written also as $M_{f, g}$. If $g=0, M_{f, g}$ will be called a input memory $F A$ and will be written simply as $M_{f}$; in this case, the memory order of $f=\beta$ is simply an integer $h$, will be denoted by $m(f)=h$.

It is clear that $M_{f, g}$ is $\tau$-weakly invertible if and only if $M_{f}$ is so, and all the problems on the weak invertibility of the separable memory FAs can be reduced to those of the input memory FAs. In order to understand the separable memory FAs, it is enough to understand the input memory FAs, so, in this paper we will mainly care about input memory FAs.

Let $\mathcal{F}$ be the set of all possible input memory FAs with $X=Y=F^{l}$ :

$$
\mathcal{F}=\left\{f \mid f=f\left(t_{-h}, \cdots, t_{-1}, t_{0}\right): X^{1+h} \rightarrow X, h \geq 0,\right\}
$$

Here $t_{-i}=\left(t_{-i, 1}, t_{-i, 2}, \cdots, t_{-i, l}\right)^{t}$, where $t$ means the transpose, and $t_{-i, j}$ is a variable taking the values from $F$.

Let $f=f\left(t_{-h}, \cdots, t_{-1}, t_{0}\right), g=g\left(t_{-h^{\prime}}, \cdots, t_{-1}, t_{0}\right) \in \mathcal{F}$. Define the product of $f$ and $g$ as

$$
f g=f\left(g\left(t_{-h-h^{\prime}}, \cdots, t_{-h}\right), \cdots, g\left(t_{-h^{\prime}}, \cdots, t_{0}\right)\right)
$$


The FA $M_{f g}$ is denoted by the notation $C^{\prime}\left(M_{g}, M_{f}\right)$ in [13]. For any state $s=\left(a_{-h-h^{\prime}} \cdots a_{-2} a_{-1}\right) \in X^{h+h^{\prime}}=S_{f g}$, it is known [13] that

$$
s \sim\left(t, s_{0}\right) \in S_{f} \times S_{g},
$$

where

$$
\begin{aligned}
s_{0} & =\left(a_{-h^{\prime}} \cdots a_{-2} a_{-1}\right) \in X^{h^{\prime}}=S_{g} \\
t & =M_{g}\left(a_{-h-h^{\prime}} \cdots a_{-h-2} a_{-h-1}\right) a_{-h} \cdots a_{-1} \in X^{h}=S_{f}
\end{aligned}
$$

hence $M_{f g}$ is a sub-automaton of $M_{f} \times M_{g}$.

With the above multiplication and the usual addition, $\mathcal{F}$ forms a quasi-ring, that is, these operations satisfy the laws of a ring except the right-distribution law.

Let $M_{m, l}(F)$ denote the set of all $m \times l$ matrices over $F$, similarly for $M_{m, l}(F[z]) \ldots$ etc. Under the mapping

$$
A=\sum_{0 \leq i \leq r} A_{i} z^{i} \mapsto \sum_{0 \leq i \leq r} A_{i} t_{-i}, \forall A \in M_{l, l}(F[z]) \text {, where } A_{i} \in M_{l, l}(F),
$$

the matrix ring $M_{l, l}(F[z])$ is embedded in $\mathcal{F}$ and becomes a subring of $\mathcal{F}$, it is exactly the set of all linear FAs in $\mathcal{F}$. $t_{0}$ is the identity of $\mathcal{F}$ and will be identified with the identity matrix $I$ and written as 1 sometimes. Similarly, $t_{-i}$ can be identified with the matrix $z^{i} I$ and written as $z^{i}$ sometimes.

More generally, let $\mathcal{F}_{m, l}$ be the set of input memory FAs whose output and input space have dimension $m$ and $l$ respectively, the set of linear FAs in $\mathcal{F}_{m, l}$ can be identified with $M_{m, l}(F[z])$. We can similarly define products of elements of $\mathcal{F}_{n, m}$ and elements of $\mathcal{F}_{m, l}$ for any $n, m, l$. In particular, elements in $\mathcal{F}_{m, l}$ can be multiplied by elements in $M_{n, m}(F[z])$ for any $n, m, l$. So the boolean expression of an element $f=f\left(t_{-h}, \cdots, t_{0}\right) \in \mathcal{F}$ can be written as:

$$
f=C T, \quad C \in M_{l, n}(F[z]), \quad T=\left(T_{1}, T_{2}, \cdots, T_{n}\right)^{t} \in \mathcal{F}_{n, l}
$$

where $T_{i}, 1 \leq i \leq n$, are distinct standard monomials, here by a standard monomial we mean a monomial of the form:

$$
\prod_{0 \leq i \leq h} \prod_{1 \leq j \leq l} t_{-i, j}^{a_{i, j}}, \quad a_{i, j} \in\{0,1\}
$$

such that there exists a $j$ such that $a_{0, j} \neq 0$, where $t_{-i, j}^{0}=1, t_{-i j}^{1}=t_{-i j j}$.

\section{Right Weak Inverses and $M_{f}$-Equations}

In this section we study the problem of the existence of the right weak inverses and the problem of solving the equation determined by the operator $M_{f}(s)$. The following Lemma 1 is critical in our studies. From Lemma 1 one may draw an analogy between a WIFA and a usual map, as it is well known that a map 
between two finite sets of the same size is injective if and only if it is surjective. To start with, we need to introduce a notion which generalizes the surjectiveness of the usual functions. For a state $s$ of $M$, we say $M(s)$ is $\tau$-surjective if

$$
M(s) X^{\tau+1}=\left(M(s) X^{\tau}\right) \times X
$$

where $M(s) X^{k}=\left\{M(s) \underline{x} \mid \underline{x} \in X^{k}\right\}, \forall k \geq 1$.

Lemma 1 Let $f \in \mathcal{F}$, then $f$ is $\tau$-weakly invertible if and only if $M_{f}(s)$ is $\tau$-surjective for all $s \in S_{f}$.

Theorem 1 Let $f \in \mathcal{F}, M^{*}=\left(X, X, S^{*}, \delta^{*}, \lambda^{*}\right)$ be a $\tau$-weak inverse of $M_{f}$. Then $M^{*}$ is also a right $\tau$-weak inverse of $f$. Moreover, if $\left(s^{*}, s\right)$ is a $\tau$-pair in $M^{*} \times M_{f}$, let $s^{* *}=\delta^{*}\left(s^{*}\right) M_{f}(s) \underline{x}^{\tau}$ for an arbitrary $\underline{x}^{\tau} \in X^{\tau}$, then $\left(s, s^{* *}\right)$ is a natural $\tau$-pair in $M_{f} \times M^{*}$.

Remark 1 Based on the above Theorem, we may concentrate only on the weak inverses.

Theorem 2 Let $f \in \mathcal{F}$ be weakly invertible with $\tau(f)=\tau$, and let $M^{*}=$ $\left(X, X, S^{*}, \delta^{*}, \lambda^{*}\right)$ be a $\tau^{\prime}$-weak inverse of $M_{f}$, and let $\left(s^{*}, s\right)$ be a $\tau^{\prime}$-pair in $M^{*} \times M_{f}$. Then

1. The $M_{f}$-equation

$$
M_{f}(s) \underline{x}=\underline{a}, \underline{a}=a_{0} a_{1} \cdots a_{n-1+\tau} \in X^{n+\tau}, \underline{x}=x_{0} x_{1} \cdots x_{n-1+\tau}, n \geq 0,
$$

has a solution $\underline{x} \in X^{\tau+n}$ if and only if $a_{0} a_{1} \cdots a_{\tau-1} \in M_{f}(s) X^{\tau}$, and if it has a solution, then the first $n$ inputs $x_{0} x_{1} \cdots x_{n-1}$ are uniquely determined.

2. If the equation (5) has a solution, then $\underline{x}$ is a solution if and only if it can be read out by applying $M^{*}\left(s^{*}\right)$ on $\underline{a} \underline{*}^{\tau^{\prime}}$ for some $\underline{*}^{\tau^{\prime}} \in X^{\tau^{\prime}}$ as follows:

$$
\underline{*}_{1}^{\tau^{\prime}} \underline{x}=M^{*}\left(s^{*}\right) \underline{a} \underline{*}^{\tau^{\prime}}
$$

where $\underline{*}_{1}^{\tau^{\prime}} \in X^{\tau^{\prime}}$ is irrelevant data.

In the sequel, a separable memory $\mathrm{FA}$ is denoted by the notation $M_{f, z g}$ naturally, where $f \in \mathcal{F}$ and $g \in \mathcal{F}$.

Theorem 3 1. For any $f \in \mathcal{F}$ and $g \in \mathcal{F}$, the equation $M_{f, z g}(s, r) \underline{x}=\underline{a}$ is equivalent to the equation $M_{f}(s) \underline{x}=\underline{a^{\prime}}, \underline{a}^{\prime}=M_{1-z g}(r) \underline{a}$.

2. Let $f \in \mathcal{F}, \tau \geq \tau(f), S_{f}=X^{h}, s^{-} \in X^{h-\tau}, \underline{a} \in X^{n}$, then the equation $M_{f}\left(s^{-} \underline{x}^{\tau}\right) \underline{x}=\underline{a}$ always has a solution $\underline{x}^{\tau} \underline{x} \in \bar{X}^{\tau+n}$. Moreover, the data $\underline{x}^{\tau} \underline{x} \in \bar{X}^{n+\tau}$ is a solution if and only if $\underline{x}^{\tau} \underline{x}$ satisfies:

$$
M_{f}\left(\underline{b}^{\tau} s^{-}\right) \underline{x}^{\tau} \underline{x}=\underline{c}^{\tau} \underline{a}
$$

for some $\underline{b} \in X^{\tau}, \underline{c}^{\tau} \in M_{f}\left(\underline{b}^{\tau} s^{-}\right) X^{\tau}$. 
3. Assume $f=f_{2} f_{1}$ and $s$ is equivalent to the state $\left(s_{2}, s_{1}\right) \in S_{f_{2}} \times S_{f_{1}}$ (see (3)). Assume $M_{2}^{*}$ is a $\tau_{2}$-weak inverse of $M_{f_{2}}$, and $\left(s_{2}^{*}, s_{2}\right)$ is a $\tau_{2}$ pair in $M_{2}^{*} \times M_{f_{2}}$. Then $\underline{x}$ is a solution of the equation (5) if and only if it satisfies:

$$
\underline{a}^{\prime}=M_{f_{1}}\left(s_{1}\right) \underline{x}
$$

where $\underline{a}^{\prime}$ is obtained as follows:

$$
\underline{*}_{1}^{\tau_{2}} \underline{a}^{\prime}=M_{2}^{*}\left(s_{2}^{*}\right) \underline{a} \underline{*}^{\tau_{2}}, \underline{*}^{\tau_{2}} \in X^{\tau_{2}}
$$

\section{Constructing WIFAs}

Denote the set consisting of all possible weakly invertible elements in $\mathcal{F}$ by $\mathcal{F}^{*}$, and denote the set consisting of all possible $\tau$-weakly invertible elements in $\mathcal{F}$ by $\mathcal{F}_{\tau}^{*}$. In this section, we study how to construct the elements in $\mathcal{F}^{*}$ and how to construct their weak inverses and the related state pairs. The last problem will be considered in Theorem 4. As will be shown, there are two types of primitive weakly invertible elements, namely weakly invertible linear FAs and 0-weakly invertible FAs, they and their weak inverses can be constructed systematically (Theorem 5 and 6 ). More elements in $\mathcal{F}^{*}$ can be generated with these two type of primitive elements by making (finite number of) the multiplicative and some proper additive operations (Theorem 7 and 8). Note that 0 -WIFAs have no contribution to the information delay in such constructions, it would be interesting if one can construct systematically nonlinear WIFAs with positive delays without using any linear FAs as ingredients, but it seems a hard task.

In the sequel we denote the group consisting of all invertible $l \times l$ matrices over $F[z]$ by $G L_{l}(F[z])$, similarly for $G L_{l}(F) \ldots$ etc.

Theorem 4 Let $M^{*}=\left(X, X, S^{*}, \delta^{*}, \lambda^{*}\right)$ be a $\tau$-weak inverse of $f \in \mathcal{F}^{*}$, given a single $\tau$-pair $\left(b^{*}, b\right)$ in $M^{*} \times M_{f}$, for any state $s \in F^{h}=S_{f}$ in $M_{f}$, let $s^{*}=\delta^{*}\left(b^{*}\right) M_{f}(b) \underline{*}^{d} s$, then $\left(s^{*}, s\right)$ is a natural $\tau$-pair in $M^{*} \times M_{f}$, where $\underline{*}^{d} \in X^{d}$, and $d=0$ if $h \geq \tau$ and $d=\tau-h$ if $\tau>h$.

Remark 2 For any given IMFA $M_{f}$ and its a $\tau$-weak inverse $M_{*}$, in order to be able to construct a $\tau$-match for each of the states in $M_{f}$, it is enough to be able to construct only a single $\tau$-pair in $M^{*} \times M_{f}$ according to the above Theorem.

In order to describe all the linear elements in $\mathcal{F}^{*}$, we need the following kind of decompositions of matrices in $M_{l, l}(F[z])$. For any $0 \neq B \in M_{l, l}(F[z])$, by using the well-known algorithm [27] for transforming a matrix over $F[z]$ into diagonal form, one can get a decomposition of $B$ of the form as below,

$$
B=P D Q(1-z b)
$$


where $P \in G L_{l}(F[z]), Q \in G L_{l}(F), b \in M_{l, l}(F[z])$ and $D$ is a $l \times l$ diagonal matrix determined by a tuple $\underline{n}=\left(n_{0}, n_{1}, \cdots, n_{\tau}\right)$ of integers

$$
\begin{array}{r}
D=\operatorname{diag}\left(I_{n_{0}}, z I_{n_{1}}, z^{2} I_{n_{2}}, \cdots, z^{\tau} I_{n_{\tau}}, 0_{n}\right), \\
n=l-\sum_{0 \leq i \leq \tau} n_{i}, \tau \geq 0, n_{\tau}>0, n_{i} \geq 0(i<\tau),
\end{array}
$$

where $I_{n}$ is the $n \times n$ identity matrix, $0_{n}$ is the $n \times n$ zero matrix. The tuple $\underline{n}$ is uniquely determined by $B$ and will be called the structure parameter of $B$.

Theorem $5[15,16,26]$ Let $B \in M_{l, l}(F[z])$ is of the form as in $(6)$, then

1. $B$ is weakly invertible if and only if $\operatorname{det}(B) \neq 0$, which is equivalent to $l=\sum_{0 \leq i \leq \tau} n_{i}$

2. If $B$ is weakly invertible, then $\tau(B)=\tau$.

3. If $\tau(B)=\tau$, then $M_{A, z b}$ is a $\tau$-weak inverse of $B$, where $A=Q^{-1} C P^{-1}$, $C=z^{\tau} D^{-1}$; and $((\underline{0}, \underline{0}), \underline{0})$ is a $\tau$-pair in $M_{A, z b} \times M_{B}$.

Theorem 6 Let $f=f\left(t_{-h}, \cdots, t_{-1}, t_{0}\right) \in \mathcal{F}$, then $f$ is 0 -weakly invertible if and only if $f\left(a_{-h}, \cdots, a_{-1}, t_{0}\right)$ is a permutation on $X$ for each state $s=$ $\left(a_{-h}, \cdots, a_{-1}\right)$ in $M_{f}$, and in this case, $f$ can be expressed as the following form:

$$
f=\sum_{1 \leq i \leq n} c_{i}\left(t_{-h}, \cdots, t_{-1}\right) P_{i}\left(t_{0}\right)
$$

where $n \geq 1, P_{i}$ is a permutation on $X$, the coefficient $c_{i}\left(t_{-h}, \cdots, t_{-1}\right)$ is a function taking the values in $\{0,1\}$ on the understanding that $0 P_{i}\left(t_{0}\right)=0$, $1 P_{i}\left(t_{0}\right)=P_{i}\left(t_{0}\right)$, and $\sum_{1 \leq i \leq n} c_{i}\left(t_{-h}, \cdots, t_{-1}\right)=1$ (as integer sum), moreover, put

$$
\beta=\sum_{1 \leq i \leq n} c_{i}\left(u_{-h}, \cdots, u_{-1}\right) P_{i}\left(t_{0}\right)^{-1}
$$

then the memory $F A M(\beta)$ is a 0 -weak inverse of $M_{f}$, it has the same state set as $M_{f}$, and $(s, s)$ is a 0 -pair in $M(\beta) \times M_{f}$ for any states in $M_{f}$. In particular, the following three types of elements are all 0 -weakly invertible: 1 . permutations on $X$; 2. $1+z k, k \in \mathcal{F}$; 3. $1+U k V$, where $U V=V U=0, U \in M_{l, l}(F[z])$, $V \in M_{l, l}(F[z])$, and $k \in \mathcal{F}$.

It is known that $\mathcal{F}^{*}$ is closed under the multiplicative operation, i.e., if $f_{i} \in \mathcal{F}^{*}(i=1,2)$, then $f_{2} f_{1} \in \mathcal{F}^{*}$. Moreover, we have

Theorem 7 Let $f_{i} \in \mathcal{F}, i=1,2$, then $f_{2} f_{1} \in \mathcal{F}^{*}$ if and only if $f_{i} \in \mathcal{F}^{*}$ for $i=1$ and 2 . And in this case $\tau\left(f_{i}\right) \leq \tau\left(f_{2} f_{1}\right) \leq \tau\left(f_{1}\right)+\tau\left(f_{2}\right)$.

To describe the inverse of the composed FA $M_{f_{2} f_{1}}$, the following construction is useful. Given $M=(X, X, S, \delta, \lambda)$, let

$$
M^{(\tau)}=\left(X, X, S \times\{0,1, \cdots, \tau\}, \delta^{(\tau)}, \lambda^{(\tau)}\right)
$$


where

$$
\delta^{(\tau)}(s, i) x= \begin{cases}(s, i+1), & 0 \leq i<\tau \\ (\delta(s, x), \tau), & i=\tau\end{cases}
$$

and

$$
\lambda^{(\tau)}(s, i) x= \begin{cases}\underline{0} \in X, & 0 \leq i<\tau \\ \lambda(s, x), & i=\tau\end{cases}
$$

The following theorem is well-known:

Theorem 8 Let $f_{i} \in \mathcal{F}, i=1,2$, and $M_{i}^{*}$ be a $\tau_{i}$-weak inverse of $M_{f_{i}}$, then $M_{1}^{*\left(\tau_{2}\right)} \times M_{2}^{*}$ is a $\left(\tau_{1}+\tau_{2}\right)$-weak inverse of $M_{f_{2} f_{1}}$. Moreover, for any state $s$ in $M_{f_{1} f_{2}}$, let $\left(s_{2}, s_{1}\right)$ be the state in $M_{f_{2}} \times M_{f_{1}}$ and equivalent to $s$ (see (3), and let $\left(s_{i}^{*}, s_{i}\right)$ be a $\tau_{i}$-pair in $M_{i}^{*} \times M_{f_{i}}$, then $\left.\left(\left(s_{1}^{*}, 0\right), s_{2}^{*}\right),\left(s_{2}, s_{1}\right)\right)$ is a $\left(\tau_{1}+\tau_{2}\right)$-pair in $\left(M_{1}^{*\left(\tau_{2}\right)} \times M_{2}^{*}\right) \times M_{f_{2} f_{1}}$.

The next result shows that $\mathcal{F}_{\tau}^{*}$ is closed under the operation adding the elements of the form $z^{\tau+1} g, g \in \mathcal{F}$. To see how the inverses of $f+z^{\tau+1} g$ is related to that of $f$, we define the circle product of $M=(X, Y, S, \delta, \lambda)$ and $M_{\beta}$ to be the FA $M \circ M_{\beta}=\left(X, Y, S \times S_{\beta}, \delta^{\circ}, \lambda^{\circ}\right)$ where $\beta=\beta\left(t_{-h}, \cdots, t_{-1}, t_{0} ; u_{-k}, \cdots, u_{-1}\right)$ is a function from $X^{h+1} \times Y^{k}$ to $Y, S_{\beta}=X^{h} \times Y^{k}$, and for any state $\left(s_{0}, r_{0}=\right.$ $\left.\left(x_{-h}, \cdots, x_{-1}, y_{-k}, \cdots, y_{-1}\right)\right) \in S \times S_{\beta}$, and any input $x_{0}$, the functions $\delta^{\circ}$ and $\lambda^{\circ}$ are defined as

$$
\begin{aligned}
& \lambda^{\circ}\left(\left(s_{0}, r_{0}\right), x_{0}\right)=\lambda\left(s_{0}, \lambda_{\beta}\left(r_{0}, x_{0}\right)\right) \\
& \delta^{\circ}\left(\left(s_{0}, r_{0}\right), x_{0}\right)=\left(\delta\left(s_{0}, \lambda_{\beta}\left(r_{0}, x_{0}\right)\right), \lambda_{\beta}\left(r_{0}, \lambda^{\circ}\left(\left(s_{0}, r_{0}\right), x_{0}\right)\right) .\right.
\end{aligned}
$$

Theorem 9 Let $f \in \mathcal{F}_{\tau}^{*}$, and $M^{*}=\left(X, X, S^{*}, \delta^{*}, \lambda^{*}\right)$ be a $\tau$-weak inverse of $M_{f}$, then

1. $f-z^{1+\tau} g \in \mathcal{F}_{\tau}^{*}$ for any $g \in \mathcal{F}$, moreover $\tau\left(f-z^{1+\tau} g\right)=\tau(f)$.

2. $M^{*} \circ M_{t_{0}, z^{\tau+1} g}$ is a $\tau$-weak inverse of $f-z^{1+\tau} g$. For any state $s$ in $f-z^{1+\tau} g$, if $\left(s^{*}, s\right)$ is a $\tau$-pair in $M^{*} \times M_{f}$, then $\left(\left(s^{*}, s\right), s\right)$ is a $\tau$-pair in $\left(M^{*} \circ M_{t_{0}, z^{1+\tau} g}\right) \times M_{f-z^{1+\tau} g}$, where $s$ is considered naturally also as both a state of $M_{t_{0}, z^{1+\tau} g}$ and a state of $M_{f}$.

\section{Brief Introduction of FAPKC}

In this section we describe the scheme FAPKC $[17,18,19,20,21]$ in terminologies developed above.

Choose two elements $f_{0}$ and $f_{1}$ in $\mathcal{F}^{*}$ whose weak inverses can be constructed easy, and let $M_{i}^{*}$ be the constructed $\tau_{i}$-weak inverse of $M_{f_{i}}, i=0,1$. Choose $g \in \mathcal{F}$. Write $f=f_{0} f_{1}, \tau=\tau_{1}+\tau_{2}, M^{*}=M_{1}^{*\left(\tau_{0}\right)} \times M_{0}^{*}$ (which is a $\tau$ weak inverse of $M_{f}$, see Theorem 8). Write $h=h_{1}+h_{2}$, where $h_{i}=m\left(f_{i}\right)$, $k=m(z g)$. Choose $(s, r) \in X^{h} \times X^{k}$ and $\left(s^{\prime}, r^{\prime}\right) \in X^{h} \times X^{k}$, let $\left.\left(s^{*}, s\right)\right)$ be a $\tau$-pair in $M^{*} \times M_{f}$ (see Theorem 8), and $\left(s^{\prime}, s^{* *}\right.$ ) be a $\tau$-pair in $M_{f} \times M^{*}$ (see Theorem 1). Write $s^{\prime}=\underline{b} s^{-}$, where $\underline{b} \in X^{\tau}, s^{-} \in X^{h-\tau}$. Let $f=C T$ be the boolean expression of $f$ (see (4)). 
The keys and the algorithm in FAPKC are as below:

Public key: $\quad C, T, g, s, r, s^{-}, r^{\prime}, \tau$.

Private key: $\quad M^{*}, s^{*}, s^{* *}$.

Encrypting: Suppose $p \in X^{n}$ is the plaintext sequence, select $\underline{x}^{\tau} \in X^{\tau}$ randomly, then the ciphertext is $\underline{c}=M_{C T, g}(s, r) \underline{p} \underline{x}^{\tau} \in X^{n+\tau}$.

Decrypting : The plaintext $\underline{p}$ can be read out from the equation $\underline{*}^{\tau} \underline{p}=$ $M^{*}\left(s^{*}\right) M_{1-z g}(r) \underline{c}$, where $\underline{*}^{\tau} \in X^{\tau}$ is irrelevant data.

Signing : Suppose $\underline{m} \in X^{n}$ is the message to be signed, select $\underline{*}^{\tau} \in X^{\tau}$ randomly, then $\underline{d}^{\tau} \underline{d}=M^{*}\left(s^{* *}\right) M_{1-z g}\left(r^{\prime}\right) \underline{m x^{\tau}}$ is the digital signature for $\underline{m}$.

Verifying signature: The receiver verifies whether $M_{C T, z g}\left(s^{-} \underline{d}^{\tau}, r^{\prime}\right) \underline{d}=\underline{m}$. The receiver accepts $\underline{d}^{\tau} \underline{d}$ as the legal signature if the equality holds, and rejects it otherwise.

Remark 3 In the proposed schemes [17, 18, 19, 20], there are some restrictions on choosing the partial state $s^{-}$. These restrictions are not necessary in order to make the algorithm work, so all these restrictions have been deleted in the above description.

Now we list the keys which are proposed in $[17,18,19,20,21]$ as follows.

Form $1[17,18]$ : $f_{0}$ is linear, $\tau\left(f_{1}\right)=0$.

Form 2 [19]: $f_{0}$ is linear, $\tau\left(f_{1}\right)>0, M_{f_{1}}$ has a weak inverse of the form $M_{A, z k}$ with $A \in M_{l, l}(F[z]), l=8$ and

$$
T=\left(t_{0,1}, t_{0,2}, \cdots, t_{0,8}, t_{0,1} t_{-1,1}, t_{0,2} t_{-1,2}, \cdots, t_{0,8} t_{-1,8}\right)^{t} .
$$

Form 3 [20]: $f_{0}$ is linear, $l=m=8, m(T)=2$ (the memory order of $T$ ), $\tau_{0}=7, \tau_{1}=8, h_{0}+h_{1} \leq 20$, but no examples for $f_{1}$ are given in [20].

Form 4 [21]: $f_{0}=B_{0} P_{0} Q_{0}, f_{1}=B_{1} P_{1} Q_{1}$ or $f_{1}=B_{1}$, where $B_{i} \in M_{l, l}(F[z])$, $Q_{i} \in M_{l, l}(F)$, each $P_{i}$ is a permutation on $X$ and is determined by a exponential function of the form $x^{2^{a}+2^{b}}$ which is defined over $G F\left(2^{l}\right)$, where $G F\left(2^{l}\right)$ is identified with $X=F^{l}$ in a natural way.

As the outer component $f_{0}$ is nonlinear, the composition $f_{0} f_{1}$ causes an exploding boolean expression, though the nonlinear degree of $f_{0}$ is just 2 . In order to keep the public key size tolerable, the parameters have to be very small. The following table is copied from [21] to illustrate the suggested parameters and the corresponding public key sizes, where $\tau_{0} \leq h_{0}=m\left(f_{0}\right), \tau_{1} \leq h_{1}=m\left(f_{1}\right)$, $N_{1}\left(N_{2}\right)$ is the corresponding public key size when $f_{1}$ is linear (nonlinear).

\begin{tabular}{|c|r|r|r|r|r|r|r|}
\hline$l$ & 7 & 7 & 5 & 5 & 3 & 3 & 3 \\
\hline$\left(h_{0}, h_{1}\right)$ & $(1,14)$ & $(7,8)$ & $(1,19)$ & $(10,10)$ & $(1,34)$ & $(10,25)$ & $(17,18)$ \\
\hline$N_{1}$ (Bits) & 8281 & 32948 & 4075 & 20950 & 1593 & 8883 & 13041 \\
\hline$N_{2}$ (Bits) & 105840 & 414512 & 29850 & 181725 & 5400 & 34560 & 51192 \\
\hline
\end{tabular}


Remark 4 In describing the basic algorithm of FAPKC3, it is stated in the section 3 of [20] that the outer component automaton of the public key is a memory finite automaton, which is not neccessarily restricted to be of the above form 3. In this paper, we consider only the latter (i.e., form 3) which is stated in the Section 4 of [20] in describing an implementation of FAPKC3, because in [20] there is neither an example nor suggested parameters for the former except the form 3. We guess it is hard to give such an example with a tolerable public key size.

It was shown that the encrypting is insecure when the key is of the form 1 in [23] and of the form 2 in [25]. It was shown in [26] that both the encrypting and signing are insecure when the key is of the form 2 without the restriction (7).

\section{Cryptanalysis on FAPKC}

In this sectin we keep the notations in the last section, and consider the following

Problem 1 How to decode the ciphertexts and how to forge the signatures without knowing the private key of FAPKC?

We will show Problem 1 can be solved for any one of the keys of the form 1-4 listed in the last section, and also for the keys of the form 2 without the restriction shown in $(7)$.

To decode the ciphertext is exactly to solve the equation $M_{f, z g}(s, r) \underline{p} \underline{x}^{\tau}=\underline{c} \in$ $X^{n+\tau}$ (where $\underline{p} \underline{x}^{\tau}$ are unknowns), which is reduced to the equation $M_{f}(s) \underline{p} \underline{x}^{\tau}=$ $\underline{c}^{\prime}$, where $\underline{c}^{\prime}=\bar{M}_{t_{0}-z g}(r) \underline{c}$ according to Theorem 3 .

To forge a signature is exactly to solve the equation $M_{f, z g}\left(s^{-} \underline{d}^{\tau}, r^{\prime}\right) \underline{d}=\underline{m}$ (where $\underline{d}^{\tau} \underline{d}$ are unknowns), which is reduced to the equation $M_{f}\left(s^{-} \underline{d}^{\tau}\right) \underline{d}=$ $\underline{m}^{\prime}, \underline{m}^{\prime}=M_{1-z g}\left(r^{\prime}\right) \underline{m}$ according to Theorem 31 ., and further to $M_{f}\left(\underline{b}^{\tau} s^{-}\right) \underline{d^{\tau}} \underline{d}=$ $\underline{c}^{\tau} \underline{m^{\prime}}$ according Theorem 32 .. Therefore Problem 1 is reduced to

Problem 2 How to solve the $M_{f}$-equation of the form (5) for $f=f_{0} f_{1}$ ?

The following theorem shows that $f$, as an arbitrary element in $\mathcal{F}^{*}$, has a routine decomposition which will be used to reduce Problem 2. We'll say two elements $f$ and $g$ in $\mathcal{F}$ are similar if there exists $G \in G L_{l}(F[z])$ such that $f=G g$, written as $f \simeq g$.

Theorem 10 Assume $f=C T \in \mathcal{F}^{*}, C \in M_{l, n}(F[z])$, then

1. Using the well-known method [27] to transform a matrix over $F[z]$ to a diagonal form, we may get

$$
C=B(I, 0) Q, B \in M_{l, l}(F[z]), \operatorname{det}(B) \neq 0, Q \in G L_{n}(F[z]),
$$


where $(I, 0)$ is a matrix of size $l \times n, I$ is the identity matrix of $l \times l$ and 0 is the zero matrix of size $l \times(n-l)$, let $f^{N}=(I, 0) Q T$, then $f=B f^{N}$, where $f^{N}$ is uniquely determined up to the similarity.

We'll call $f^{N}$ the T-nonlinear factor of $f$, and call $B$ the T-linear factor of $f$.

2. For any weakly invertible linear $A \in \mathcal{F}$, denote the $T$-nonlinear factor of Af by $(A f)^{N}$, then $(A f)^{N} \simeq f^{N}$ and $\tau\left((A f)^{N}\right)=\tau\left(f^{N}\right) \leq \tau(f)$.

From Theorem 10 and Theorem 7 we get

Corollary 1 Let $f^{N}$ be the T-nonlinear factor of $f$ defined as in Theorem 10 , then $\tau\left(f^{N}\right) \leq \tau_{1}$ for any one of the keys of the forms 1-4 listed in the last section, and also for the keys of the form 2 without the restriction shown in (7).

Notice that the weak inverses of the linear factor of $f$ is easy constructed (see theorem 5), so basing on Theorem 10 and Theorem 33 ., Problem 2 is reduced to

Problem 3 How to solve the equation of the form $M_{f^{N}}(s) \underline{x}=\underline{a}, \underline{a} \in X^{n+\tau}$, $n \geq 1$ (where $f^{N}$ is the T-nonlinear factor of $f$ defined as in Theorem 10)?

One may try to solve Problem 3 case by case by means of the divide-andconquer searching method, or according to Theorem 2 try to solve it systematically by solving the following

Problem 4 How to construct a $\tau^{\prime}$-weak inverse of $M_{f}$ or $M_{f^{N}}$ (where $\tau^{\prime}$ can be chosen arbitrarily)?

Problem 4 can be solved if we can decompose $f$ or $f^{N}$ into a product of several FAs each of which can be inverted. It is the case when the key is of the form 2 without the restriction (7), as shown in the following theorem, which characterizes the so-called quasi-linear elements defined as below.

Definition 1 The element $f$ in $\mathcal{F}$ is called quasi-linear if $M_{f}$ has a weak inverse of the form $M_{A, z k}$ with $A \in M_{l, l}(F[z]), k \in \mathcal{F}$.

Theorem 11 Let $f \in \mathcal{F}^{*}$, then

1. $f$ is quasi-linear if and only if $f$ has a decomposition:

$$
f=B(1-z g), B \in M_{l, l}(F[z]), \operatorname{det}(B) \neq 0, g \in \mathcal{F}
$$

As a consequence, if $f$ is quasi-linear, so is $A f$ for any $A \in M_{l, l}(F[z])$, $\operatorname{det}(A) \neq 0$. 
2. If $f$ is quasi-linear, then its a decomposition $f=B(1-z g)$ of the form (9) and its a weak inverse can be obtained easy from its boolean expression $f=$ $C T$ as follows. Assume $T=\left(\begin{array}{c}t_{0} \\ T^{\prime}\end{array}\right), C \in M_{l, n}(F[z])$, correspondingly write $C=\left(C_{0}, C^{\prime}\right), C_{0} \in M_{l, l}(F[z]), C^{\prime} \in M_{l, n-l}(F[z])$. Let $C_{0}=P D Q(I-z b)$ be a decomposition of $C_{0}$ of the form (6), and let $A=z^{\tau} Q^{-1} D^{-1} P^{-1}$, then $A \in M_{l, l}(F[z])$ and $A C^{\prime}=z^{\tau+1} H$ for some $H \in M_{l, n-l}(F[z])$. Let $g=b-H T^{\prime}, B=P D Q$. Then $f=B(1-z g)$, and $M_{A, z g}$ is a $\tau$-weak inverse of $M_{f}$.

We claim that Problem 3 can be solved case by case practically by means of the divide-and-conquer searching method when the key is any one of the form $1-4$ listed in the last section. To see this, we consider how large $l \tau(f)$ should be in order to resist the devide-and-conquer searching attacks on the equation of the form $M_{f}(s) \underline{x}^{n+\tau}=\underline{a}, \underline{a} \in X^{n+\tau}$. Let's see at first how to estimate the actual complexity of such an attack. For plain exhaustive searching, an obvious upper bound is $2^{l(1+\tau(f))}$, but the exact bound may be much smaller. When $f$ is linear, the logarithm of the bound to base 2 can be expressed by its structure parameters defined in section 5, and the mean value for this expression is $\frac{l(1+\tau(f))}{2}$. There are no strong reasons why exhaustive searching with a nonlinear FA should be much harder than with a linear one. So, that we use $2^{l(1+\tau(f)) / 2}$ to estimate the complexity of the devide-and-conquer searching type attacks is not too pessimistic. Thus to resist such attacks to Problem 3 , we should require, say, $\frac{l\left(1+\tau\left(f^{N}\right)\right)}{2}>60$. Basing on Corollary 1 , the parameter $\frac{l\left(1+\tau\left(f^{N}\right)\right)}{2}$ for any one of the keys of the forms 1-3, and for any one of the suggested keys of the form 4 is estimated as below, and one can see that non of them meets the bound 60 .

1. When the key is of the form 1 and the form $2, \frac{l\left(1+\tau\left(f^{N}\right)\right)}{2}=4$.

2. When the key is of the form $3, \frac{l\left(1+\tau\left(f^{N}\right)\right)}{2}=36$

3. When the key is of the form 4, the parameter $\frac{l\left(1+\tau\left(f^{N}\right)\right)}{2}$ is shown in the following table for the suggested parameters.

\begin{tabular}{|c|c|c|c|c|c|c|c|}
\hline$l$ & 7 & 7 & 5 & 5 & 3 & 3 & 3 \\
\hline$\left(h_{0}, h_{1}\right)$ & $(1,14)$ & $(7,8)$ & $(1,19)$ & $(10,10)$ & $(1,34)$ & $(10,25)$ & $(17,18)$ \\
\hline$\frac{l\left(1+\tau\left(f^{N}\right)\right)}{2}$ & 52.5 & 31.5 & 50 & 27.5 & 52.5 & 39 & 28.5 \\
\hline
\end{tabular}

From the above cryptanalysis of this section, we see all the keys proposed in $[17,18,19,20,21]$ for FAPKC are insecure both in encrypting and signing.

\section{References}

[1] Huffman D.A., Canonical Forms for Information Lossless Finite State Logical Machines, IRE Transaction on Circuit Theory, IRE Trans. Cir. Theory, special supplement, 1959, pp.41-59. 
[2] Massey J.L. and Sain M.K., Inverses of Linear Sequential Circuits, IEEE Trans. Comput., 1968, 17: pp.330-337.

[3] Massey J.L. and Sain M.K., A modified Inverse for Linear Dynamical Systems, Proc. IEEE 8th Adaptive Processes Symp., 1969, pp. 5a1-5a3.

[4] Massey J.L. and Sain M.K., IEEE Trans. AC-14, No.2, 1969, pp.141-149.

[5] Forney G.D., Convolution Codes I: Algebraic Structures, IEEE Trans. I.T., 1970, 16: pp.720-738.

[6] Tao R.J., Invertible Linear Finite Automata, Scientia Sinica, 1973, 16: pp.565-581.

[7] Kyimit A.A., Information Lossless Automata of Finite Order, New York: Wiley, 1974.

[8] Tao R.J., Invertibility of Finite Automata (in Chinese), Beijing, Science Press, 1979: pp.39-42,68.

[9] Tao R.J., Invertibility of Linear Finite Automata over a Ring, Automata, Languages and Programming (Edited by Timo Lepisto, Arto Salomaa), Lecture Notes in Computer Sciences, Springer Verlag, 1988, 317:pp.489501.

[10] Lai X. and Massey J.L., Some Connections between Scramblers and Invertible Automata, Proc. 1988 Beijing Int. Workshop on Info.Th., 1988, pp. DI5.1-DI5.5.

[11] Juhani Heino, Finite Automata: a layman approach, text posted in sci,cript newsgroup, October 1994, Juhanihe@waltari.helsinki.fi, University of Helsinki, Finland, 1994.

[12] Tao R.J., Generating a kind of nonlinear finite automata with invertibility by transformation method, Laboratory for Computer Science, Institute of Software, Chinese Academy of Sciences, Beijing 100080, China, ISCASLCS $-95-05$.

[13] Tao R.J., On invertibility of some compound finite automata, Laboratory for Computer Science, Institute of Software, Chinese Academy of Sciences, Beijing 100080, China, ISCAS-LCS-95-06.

[14] Dai.Z.D., Invariants and Inversibility of Linear Finite Automata, Advances in Cryptology-ChinaCrypt'94 (In Chinease), Science Press, pp.127-134.

[15] Dai Z.D., Ye D.F., Weak Invertibility of Linear Finite Automata over Commutative Rings -Classification and Enumeration (in Chinese), KEXUE TONGBAO(Bulletin of Science), Vol.4, No.15, 8,1995, pp.1357-1360. 
[16] Dai Z.D., Ye D.F., Weak Invertibility of Linear Finite Automata I, Classification and Enumeration of Transfer Functions, SCIENCE IN CHINA (Series A), Vol. 39, No. 6, June 1996, pp.613-623.

[17] Tao R.C. and Chen S.H., A Finite Automaton Public Key Cryptosystem and Digital Signatures, Chinese J. of Computer, 1985(8), pp.401-409 (in Chinese).

[18] Tao R.J. and Chen S.H., Two Varieties of Finite Automaton Public Key Cryptosystem and Digital Signatures, J. of Compt. Sci. and Tech., 1986(1), No.1, pp.9-18.

[19] Tao R..., Conference report, ChinaCrypt'92, Xian, 1992.

[20] Tao R.J. and Chen S.H. and Chen X.M., FAPKC3: a new finite automaton public key cryptosystem, Laboratory for Computer Science, Institute of Software, Chinese Academy of Sciences, Beijing 100080, China, June, 1995. ISCAS-LCS-95-07.

[21] Chen X.M., The Invertibility Theory and Application of Quadratic Finite Automata, Laboratory for Computer Science, Institute of Software, Chinese Academy of Sciences, Beijing 100080, China, November,1996, Doctoral thesis.

[22] Schneier B., Applied Cryptography, second addition, 1994.

[23] Dai D.W. Wu K. and Zhang H.G., Cryptanalysis on a Finite Automaton Public Key Cryptosystem, Science in China, 1994 (in Chinese).

[24] Bao, F., Igarashi,Y., Break Finite Automata Public Key Cryptosystem, Automata, Languages and Programming, Lecture Notes in Computer Sciences, 944(1995), Springer,147-158.

[25] Qin Z.P., Zhang H.G., Cryptanalysis of Finite Automaton Public Key Cryptosystems (in Chinese), -Chinacrypt'96, Science Press, pp.75-86.

[26] Dai Z.D., A Class of Seperable Memory Finite Automata-Cryptoanalysis on FAPKC -Chinacrypt'96, Science Press, pp.75-86.

[27] Jacobson N., Basic Algebra I, W.H.Freeman and Company, San Francisco, pp.175-179.

[28] Wan Z.X., Algebra and Coding Theory (in Chinese), Beijing, Science Press, 1979 , p.510. 